\title{
Relações entre Crescimento Econômico, Exportações e lnvestimento Direto Estrangeiro no Brasil
}

\section{Relations among Economic Growth, Exports and Foreign Direct Investment in Brazil}

\author{
Lora dos Anjos Rodrigues* \\ Mateus de Carvalho Reis Neves** \\ Leonardo Bornacki de Mattos***
}

Resumo: Neste artigo, examina-se a relação causal entre crescimento econômico (PIB), exportações e investimento direto estrangeiro (IDE) no Brasil durante o período que compreende os anos de 1947 a 2010. Para tanto, estimou-se um modelo VAR, a partir do qual foi realizado o teste de causalidade de Toda e Yamamoto entre as variáveis em estudo. A análise dos resultados evidencia uma relação unidirecional entre PIB e EXP, bem como entre EXP e IDE, indicando que as exportações podem ser consideradas um "mecanismo de efeito" entre o crescimento econômico e os investimentos diretos estrangeiros. Tais resultados convergem para a importância dos investimentos em infraestrutura, dentre outros, como promotores do desenvolvimento nacional.

Palavras-chave: Crescimento econômico. Exportações. Investimento Direto Estrangeiro.

\begin{abstract}
This paper examines the causal relationship among economic growth (GDP), exports and foreign direct investment (FDI) in Brazil during the years of 1947 to 2010. The empirical study is based on a VAR, in which was applied the Toda e Yamamoto test. The results show an one-way relation between GPD and exports, as well as between exports and FDI, indicating that exports can be considered a "mechanism of effect" between economic growth and foreign direct investment. These results indicate the relevance of infrastructure investments, among others, as national development promoters.
\end{abstract}

Keywords: Economic growth. Exports. Foreign Direct Investment.

JEL Classification: F14; F21.

* $\quad$ Doutoranda em Economia Aplicada na Universidade Federal de Viçosa (UFV). Bolsista Capes. E-mail: lora_anjos@yahoo.com.br

** Mestrando em Economia Aplicada na Universidade Federal de Viçosa (UFV). Bolsista Capes. E-mail: mateuscrneves@gmail.com

*** Professor adjunto no Departamento de Economia Rural da UFV. Bolsista do Programa Funarpex da Fundação Arthur Bernades (Funarbe). E-mail: lbmattos@ufv.br 


\section{1 lntrodução}

Após o término dos anos 1980, década considerada "perdida", em que o Brasil atingiu taxas ínfimas de crescimento econômico, em um contexto de altas taxas de inflação e restrição ao capital externo, o país iniciou os anos 1990 sob outra perspectiva.

Com o advento do Plano Real, em 1994, a estabilização econômica foi acompanhada por uma entrada de Investimento Direto Estrangeiro (IDE) sem precedentes. Segundo Fernandes (2006), no início da década de 1990, o estoque de IDE no Brasil era da ordem de US $\$ 37.243$ bilhões, passando para US $\$ 103.015$ bilhões em 2000, quando atingiu seu auge. Seguindo a tendência que se configurava no cenário internacional, de elevação do fluxo de capitais, o país destacou-se como uma das principais economias receptoras de IDE, ocupando em 1996, conforme Matesco et al. (2000), a sétima posição no ranking mundial. Adicionalmente, o Programa Nacional de Desestatização (PND), iniciado em 1990, contribuiu de forma significativa para atração destes investimentos.

Caracterizada também pela abertura comercial, a década de 1990 assistiu à recuperação do desempenho das exportações brasileiras, onde se iniciou a superação do viés antiexportador que, segundo Bonelli e Pinheiro (2008), foi resultante do Processo de Substituição de Importações, iniciado em 1930. Entre 1991 e 2008, as exportações cresceram em valor e quantidade (SILVA; LIMA; BEZERRA, 2010). Embora, a partir de 1995, a Balança Comercial tenha se apresentado deficitária, em 2002 voltou a mostrar saldo positivo, batendo sucessivos recordes e, segundo Focus/BCB (2007), superando as expectativas do mercado.

O comércio exterior brasileiro teve sua dinâmica e padrão fortemente influenciados pelas empresas transnacionais. De 1995 a 2000, a participação das empresas estrangeiras no total das exportações passou de $46,8 \%$ para $60,4 \%$ (LACERDA, 2004). Cabe ressaltar que as importações também cresceram significativamente neste período.

Também nos anos 1990, a taxa de crescimento econômico iniciou uma lenta, mas progressiva recuperação. De acordo com dados do Banco Central do Brasil (BCB) (2010), a taxa real média de crescimento anual do Produto Interno Bruto (PIB), que foi de $1,6 \%$, na década de 1980 , sofreu uma pequena elevação de $1,8 \%$ nos anos 1990 e avançou para 3,2\% entre 2000 e 2009.

Houve, portanto, a partir da década de 1990, início da recuperação dos níveis de IDE, exportações e taxa de crescimento econômico. Visto que há um relacionamento complexo, fundamentado teoricamente, sobre o modo como estas variáveis interagem entre si, objetiva-se com este estudo, verificar empiricamente as relações de interdependência entre PIB, exportações e IDE na economia brasileira no período de 1947 a 2010. 
Os estudos realizados especificamente para o Brasil caracterizaram as relações de somente duas destas variáveis simultaneamente ${ }^{1}$. De maneira geral, há estudos que procuraram evidências sobre teorias como export-led growth e/ou growth-driven export, por meio da verificação de causalidade entre exportações e crescimento econômico. Alguns procuraram, ainda, verificar se a significativa entrada de IDE na década de 1990 foi determinante para o crescimento econômico, enquanto outros buscaram relacionar estes investimentos ao nível de exportações.

Assim, dada a existência de um debate teórico e ausência de consenso nas evidências empíricas sobre as relações entre essas variáveis, este estudo contribui com a literatura ao verificar empiricamente se alguma das variáveis estudadas se comporta como "mecanismo de efeito" 2 no período analisado. Portanto, ao relacionar as três variáveis em um único modelo, é possível verificar se uma variável afeta outra de forma indireta, por meio de seus efeitos sobre uma terceira variável, o que a configuraria como "mecanismo de efeito" ou intermediária.

O artigo está estruturado em outras cinco seções, além desta introdução. $\mathrm{Na}$ segunda seção apresenta-se a discussão teórica. A seguir, apresentam-se algumas evidências empíricas que fundamentam a pesquisa. Na quarta seção descreve-se o método e os dados utilizados. Na quinta seção expõem-se os resultados e, por fim, a última seção contém a conclusão do artigo, com alguns comentários relevantes.

\section{Referencial Teórico}

Existem teorias bem fundamentadas sobre o relacionamento entre exportações, crescimento econômico e IDE.

A discussão sobre os efeitos do comércio internacional remonta a Adam Smith, com sua teoria das vantagens absolutas. Ao lado de outros modelos tradicionais, como as "vantagens comparativas" de Ricardo, modelos Hecksher-Ohlin (H-O) e sua versão dinâmica, Hecksher-Ohlin-Samuelson (H-O-S), discorre sobre os benefícios do comércio, acerca dos ganhos de bem-estar e crescimento econômico entre as nações envolvidas, por meio da especialização na produção. No entanto, segundo Jayme Júnior (2001), os pressupostos restritivos não permitem a validação empírica dos modelos.

Contrariamente aos modelos clássicos, fundamentados no princípio da Lei de Say, pós-Keynesianos formularam teorias sobre o comércio internacional, sob a ótica de que é nos componentes da demanda agregada que se encontram os determinantes do crescimento. Kaldor (1970) formulou o modelo export-led growth, de crescimento liderado pelas exportações, em que há um mecanismo de atuação

$1 \quad$ Os estudos serão apresentados na seção destinada à revisão de literatura.

2 O termo "mecanismo de efeito" se refere a uma variável que se comporta como um canal pelo qual outra variável afeta uma terceira indiretamente. Na relação $a \rightarrow b \rightarrow c$, por exemplo, $b$ é o canal pelo qual a afeta $c$. 
cumulativo: estímulos à exportação resultam em crescimento do PIB, pois o aumento das exportações leva ao aumento da produtividade do fator devido a ganhos de escala, resultantes do mercado externo maior. Quanto maior a taxa de crescimento do PIB, maior a taxa de produtividade e, assim, menores os custos unitários, de modo que maiores serão as exportações.

É neste último sentido que discorre a teoria growth-driven export, de crescimento econômico induzindo as exportações, em que o crescimento experimentado por uma economia, resultante de um processo de acumulação de capital físico e/ou transferência de tecnologia, via IDE, pode induzir aumentos das exportações, quando o mercado doméstico não absorve totalmente o aumento na produção de bens.

O modelo de Solow (1956) é um dos pioneiros a evidenciar que a acumulação de capital físico e o progresso tecnológico são as forças motoras do crescimento econômico. Dos trabalhos de Romer (1986) e Lucas (1988) "nasceu" a Teoria do Crescimento Endógeno. Segundo esta, por meio de spillovers e externalidades, o IDE afeta a taxa de crescimento pela incorporação de novos insumos e novas tecnologias na função de produção do país receptor.

Sob a perspectiva Keynesiana, o produto potencial é determinado endogenamente, visto que o estoque de capital é apontado conforme as expectativas de demanda dos empresários, que serão mais otimistas quanto mais acelerado for o crescimento, estimulando a acumulação de capital que, por sua vez, promove crescimento. Assim, o IDE realizado por corporações multinacionais é atraído por economias produtivas e em crescimento.

O IDE também pode exercer influência positiva sobre o comércio exterior. Segundo Dunning (1988), os investimentos do tipo resource seeking geram pouco vínculo com o país receptor, pois, na medida em que visam aquisição de recursos, a baixos custos, para produção de bens, contribuem para aumentar os fluxos de exportação. No mesmo sentido, Rugman et al. (1985), reorganizando o modelo de Hirsh (1976), enunciam que empresas que realizam IDE tem como alvo o mercado do próprio país de origem, elevando assim o nível de exportações do país hospedeiro. Outra forma pela qual o IDE contribui para o aumento no volume exportado, segundo Buckley e Casson (1976; 1981), é quando estes investimentos têm por objetivo a internalização de custos de transação, buscando minimizá-los. Adicionalmente, Keesing e Lall (1992), afirmam que nos países em desenvolvimento, a maioria das empresas domésticas não tem habilidade e recursos para obter êxito no comércio internacional.

Já Grossman e Helpman (1991) acreditam que é o livre comércio que contribui para melhoria do clima para investimentos. 
Portanto, há um debate teórico quanto às relações entre exportações, crescimento econômico e investimento direto estrangeiro (IDE), pois as abordagens consideram condições e pressupostos diversos.

\section{Revisão de Literatura}

A ausência de evidências empíricas conclusivas contribui para o acirramento do debate sobre a relação entre exportações, crescimento econômico e IDE.

Kónya (2000) verificou, no Reino Unido e Suécia, relação bi-causal entre exportações e PIB e confirmou ambas as teorias, de export-led growth e growthdriven export, enquanto no Japão e Canadá, observou que o crescimento induziu as exportações, no período de 1960 a 1998. No entanto, Awokuse (2003) utilizouse de dados trimestrais, entre 1961 a 2004, e encontrou resultado diverso para a economia canadense, sendo as exportações precedentes ao crescimento. No estudo realizado por Chow (1987), que incluiu oito países em desenvolvimento, no período de 1960 a 1987, verificou-se ausência de causalidade entre exportações e crescimento na Argentina, causalidade das exportações para o crescimento no México e, no caso do Brasil e demais países, causalidade bidirecional.

Especificamente para o Brasil, Matos (2003) também encontrou bicausalidade entre exportações e crescimento, entre 1980 e 2002, período posterior ao analisado por Chow (1987). Fochezatto, Koshiyama e Alencastro (2010) utilizaram dados anuais, de 1947 a 2006, e diversas especificações do modelo VAR para aplicar o teste de causalidade de Toda e Yamamoto, com o qual concluíram que as exportações causaram positivamente o PIB. Silva, Virgolino e Lima (2000) não encontraram relação de longo prazo entre estas variáveis na economia brasileira. Fasano Filho (1988) constatou a hipótese de export-led growth na indústria manufatureira. Tyler (1982) também verificou que as exportações contribuíram para o crescimento do produto na indústria de transformação brasileira.

De modo semelhante, os trabalhos empíricos também não convergiram em seus resultados para as relações entre IDE e crescimento. Borenstein, Gregorio e Lee (1995) analisaram a causalidade em países desenvolvidos e em desenvolvimento, no período de 1970 a 1989, e concluíram que o IDE tem efeito positivo sobre o crescimento econômico. Igualmente, Buckley et al. (2002) observaram que a taxa de crescimento do IDE afetou positivamente o crescimento do PIB para diversas regiões da China, no período 1989 a 1998. Nonnenberg e Mendonça (2005) encontraram relação no sentido inverso, em que PIB afetou IDE, em alguns países em desenvolvimento, incluindo o Brasil, entre 1975 e 2000. Por outro lado, em uma análise somente de dados para a economia brasileira, Fernandes (2006) não encontrou relação de causalidade entre PIB per capita e IDE, por meio do teste de Toda e Yamamoto, no período 1970 a 2003. 
Por fim, ao analisar relações causais entre IDE e exportações para China e algumas de suas províncias, Zhang e Felminghan (2001) confirmaram que estas relações diferiram conforme o volume de investimento recebido por elas no período de 1986 a 1999. Liu et al. (2001) verificaram que IDE causou, no sentido de Granger, exportações na China, no período de 1984 a $1998^{3}$.

Adicionalmente, na literatura internacional já existem alguns estudos que relacionam PIB, exportações e IDE em um único modelo, para verificar a existência de causalidade no sentido de Granger, por meio de um possível "mecanismo de efeito". Hsiao e Hsiao (2006) estimaram um modelo VAR para oito economias da Ásia, entre 1986 e 2004, e verificaram que, em geral, IDE afeta o PIB indiretamente, por meio de seus impactos sobre as exportações, que acabaram por funcionar como "mecanismo de efeito". Miankhel, Thangavelu e Kalirajan (2009) por meio da abordagem VAR multivariado, verificaram a dinâmica de relacionamento entre estas três variáveis com o teste de Granger para alguns países emergentes em diferentes estágios de crescimento. Seus resultados indicam que, por exemplo, no Chile, o investimento direto estrangeiro causa exportação de forma direta e indireta por meio de seus efeitos no PIB, sendo este um "mecanismo de efeito". Dritsaki, Dritsaki e Adamopoulos (2004) encontraram causalidade bidirecional entre PIB e exportações, unidirecional do IDE para exportações e do IDE para o PIB, para a Grécia, com dados anuais de 1960 a 2002. Nair-Reichert e Weinhold (2000), com dados de 1971 a 1995, para 24 países em desenvolvimento, observaram que IDE causa PIB. Cho (2005) constatou somente a existência de causalidade unidirecional do IDE para as exportações para China, Taiwan, Coreia do Sul, Hong Kong, Singapura, Malásia, Tailândia, Indonésia e Filipinas, entre 1970 e 2001.

É importante notar que, em análises de causalidade, os resultados variam de acordo com o método empregado, o país ou região estudados, período em análise, tratamento das variáveis, bem como a presença de variáveis de controle no modelo.

\section{Metodologia}

Com o objetivo de verificar as relações de interdependência e a direção da causalidade, no sentido de precedência temporal, entre as variáveis crescimento econômico, exportações e investimento direto estrangeiro, foi adotado o procedimento de Toda e Yamamoto (1995), que consiste no uso direto dos estimadores de mínimos quadrados ordinários (MQO) na resolução de um sistema vetorial autorregressivo (VAR) aumentado.

3 Relativamente aos trabalhos que relacionam $P I B$ e exportações, são escassos aqueles que analisam causalidade no sentido de Granger entre PIB e IDE e; IDE e exportações, para o Brasil. 
Outra possibilidade seria a utilização do teste de causalidade de Granger (1969), que carece da realização de pré-testes para verificar a existência de raiz unitária e cointegração (caso haja raiz unitária) no modelo VAR a ser estimado. Entretanto, os testes de cointegração, além de pouco robustos, permitem diversas especificações, possibilitando que ocorra arbitrariedade em sua definição. A utilização do método de Toda e Yamamoto (1995) não requer testes de cointegração e, portanto, contorna estes problemas, permitindo-se aplicar a sistemas cujas variáveis possuam diferentes ordens de integração e que sejam cointegrados. Além disso, experimentos realizados por Zapata e Rambaldi (1997) demonstraram que, em amostras com tamanho próximo a 50 observações, o teste de Toda e Yamamoto possui desempenho parecido ao de outros métodos mais complexos.

Tal teste consiste, essencialmente, em se colocar restrições aos parâmetros, por meio do teste de Wald, de um modelo VAR $(k+d)$, em que $k$ é o número de defasagens e $d$ a ordem de integração do sistema. Daí advém o emprego do termo VAR aumentado. Este teste possui distribuição assintótica qui-quadrado.

\subsection{Modelo Empírico}

A opção pela abordagem VAR deve-se ao fato de se pressupor que todas as variáveis são endógenas, eliminando, assim, a possibilidade do viés de simultaneidade ${ }^{4}$.

Segundo Fochezatto, Koshiyama e Alencastro (2010), pode-se especificar um modelo VAR em níveis com $(k+d)$ defasagens da seguinte forma:

$$
\begin{aligned}
& P I B_{t}=\Phi_{1}+\sum_{i=1}^{k} \beta_{1 \mathrm{i}} \mathrm{PIB}_{\mathrm{t}-\mathrm{i}}+\sum_{\mathrm{j}=\mathrm{k}+1}^{k+d} \beta_{1 j} \mathrm{PIB}_{\mathrm{t}-\mathrm{j}}+\sum_{i=1}^{k} \gamma_{1 i} \mathrm{EXP}_{\mathrm{t}-\mathrm{i}}+\sum_{\mathrm{j}=\mathrm{k}+1}^{k+d} \gamma_{1 j} \mathrm{EXP}_{\mathrm{t} \cdot \mathrm{j}} \\
& +\sum_{\mathrm{i}=1}^{k} \delta_{1 i} \mathrm{IDE}_{\mathrm{t}-\mathrm{i}}+\sum_{\mathrm{j}=\mathrm{k}+1}^{k+d} \delta_{1 j} \mathrm{IDE}_{\mathrm{t}-\mathrm{j}}+\varphi_{1} \mathrm{DUMMY}+e_{1 \mathrm{t}} \\
& E X P_{t}=\Phi_{2}+\sum_{i=1}^{k} \beta_{2 \mathrm{i}} \mathrm{PIB}_{\mathrm{t}-\mathrm{i}}+\sum_{\mathrm{j}=\mathrm{k}+1}^{k+d} \beta_{2 j} \mathrm{PIB}_{\mathrm{t} \cdot \mathrm{j}}+\sum_{i=1}^{k} \mathrm{\gamma}_{2 i} \mathrm{EXP}_{\mathrm{t}-\mathrm{i}}+\sum_{\mathrm{j}=\mathrm{k}+1}^{k+d} \mathrm{\gamma}_{2 j} \mathrm{EXP}_{\mathrm{t} \cdot \mathrm{j}} \\
& +\sum_{\mathrm{i}=1}^{k} \delta_{2 i} \mathrm{IDE}_{\mathrm{t} \mathrm{i}}+\sum_{\mathrm{j}=\mathrm{k}+1}^{k+d} \delta_{2 j} \mathrm{IDE}_{\mathrm{t} \mathrm{j}}+\varphi_{1} \mathrm{DUMMY}+e_{2 t} \\
& I D E_{t}=\Phi_{3}+\sum_{i=1}^{k} \beta_{3 \mathrm{i}} \mathrm{PIB}_{\mathrm{t}-i}+\sum_{\mathrm{j}=\mathrm{k}+1}^{k+d} \beta_{3 j} \mathrm{PIB}_{\mathrm{t}-\mathrm{j}}+\sum_{i=1}^{k} \gamma_{3 i} \mathrm{EXP}_{\mathrm{t}-\mathrm{i}}+\sum_{\mathrm{j}=\mathrm{k}+1}^{k+d} \gamma_{3 j} \mathrm{EXP}_{\mathrm{t}-\mathrm{j}} \\
& +\sum_{\mathrm{i}=1}^{k} \delta_{3 i} \mathrm{IDE}_{\mathrm{t} \cdot \mathrm{i}}+\sum_{\mathrm{j}=\mathrm{k}+1}^{k+d} \delta_{3 j} \mathrm{IDE}_{\mathrm{t} \cdot \mathrm{j}}+\varphi_{1} \text { Dummy }+e_{3 t}
\end{aligned}
$$

4 Para mais detalhes acerca do modelo VAR, ver Bueno (2008). 
em que PIB representa o Produto Interno Bruto, EXP refere-se às Exportações, IDE é o Investimento Direto Estrangeiro, $\mathrm{k}$ corresponde ao número de defasagens, $d$ é a ordem de integração do sistema e $e_{n t}$ é o termo de erro estocástico da equação $n$ no tempo $t$.

Uma variável DUMMY de intercepto foi inserida na estimação do modelo de modo a captar a significativa entrada de IDE no período pós-abertura comercial, sendo atribuído o valor zero aos anos de 1947 a 1989 e o valor um aos de 1990 a 2010. Deste modo, espera-se que o coeficiente da variável dummy seja positivo.

Após a estimação do modelo VAR mais adequado, será analisada a causalidade de Toda e Yamamoto. O teste de Wald é aplicado para testar a hipótese de não causalidade nos primeiros $k$ coeficientes. Assim, haverá, por exemplo, causalidade unidirecional de EXP para PIB se a hipótese $\mathrm{H}_{0}: \gamma_{1 i}=0$ for rejeitada e, ao mesmo tempo, $\mathrm{H}_{0}: \beta_{2 \mathrm{i}}=0$ não for rejeitada (FOCHEZATTO; KOSHIYAMA; ALENCASTRO, 2010).

\subsection{Dados}

Neste modelo, utilizam-se dados anuais de 1947 a 2010. A escolha desta periodicidade deve-se ao fato de tentar reproduzir de maneira mais próxima a dinâmica real do comportamento das variáveis utilizadas. A escolha do período visa obter maior número de observações e, por conseguinte, maior robustez dos resultados e, abarcar a significativa entrada de IDE a partir de 1989. O Quadro 1 sintetiza a descrição e as fontes destas variáveis.

Quadro 1- Descrição das variáveis utilizadas

\begin{tabular}{|c|c|c|c|}
\hline Variável & Descrição & Unidade & Fonte \\
\hline PIB & Produto interno bruto & $\mathrm{R} \$$ (milhões) & IBGE \\
\hline EXP & Exportações de bens e serviços & $\mathrm{R} \$$ (milhões) & IBGE \\
\hline$I D E$ & Investimentos diretos estrangeiros no país & $\mathrm{US} \$$ (milhões) & $\mathrm{BCB}$ \\
\hline
\end{tabular}

Fonte: Elaboração própria.

Nota: Instituto Brasileiro de Geografia e Estatística (IBGE); Banco Central do Brasil (BCB).

Inicialmente, converteu-se a variável IDE para milhões de reais pela multiplicação de seu valor expresso em dólares pela taxa de câmbio corrente. Depois, operou-se o deflacionamento das séries com 2010 como ano-base. A variável PIB (proxy para crescimento econômico) foi deflacionada pelo Deflator Implícito do PIB - índice encadeado, do IBGE, enquanto as variáveis EXP e IDE foram deflacionadas por meio do Índice Geral de Preços - Disponibilidade Interna (IGP-DI), da Fundação Getúlio Vargas (FGV). Por fim, todas as variáveis foram transformadas 
em taxa de variação, assim, as inferências realizadas no decorrer do estudo sobre $P I B, I D E$ e EXP serão relativas à taxa de crescimento destas variáveis.

\section{Resultados}

Tendo em vista o procedimento indicado na metodologia, primeiramente, realizou-se o teste de Dickey-Fuller aumentado (ADF) para verificar a existência de raiz unitária em cada uma das três séries. Conforme resultados da Tabela 1, podese verificar que todas as séries são estacionárias [I(0)] em nível, utilizando 1\% de significância, não havendo necessidade de aplicar diferenciação nas séries.

Tabela 1 - Teste de Raiz Unitária (ADF) nas séries PIB, EXPe IDE, 1947-2010

\begin{tabular}{cccccc}
\hline Série & Equação de teste & Defasagens & $\begin{array}{c}\text { Estatística } \\
\text { de teste }\end{array}$ & \multicolumn{2}{c}{ Valor Crítico } \\
$\mathbf{1} \%$ & $\mathbf{5 \%}$ \\
\hline PIB & Constante e tendência & 0 & $-5,0356 * * *$ & $-4,1130$ & $-3,4839$ \\
EXP & Constante & 0 & $-7,3159 * * *$ & $-3,5402$ & $-2,9092$ \\
IDE & Constante & 0 & $-7,2607 * * *$ & $-3,5402$ & $-2,9092$ \\
\hline
\end{tabular}

Fonte: Elaboração própria a partir dos resultados da pesquisa.

Nota: Utilizou-se o número de defasagens que minimizou o Critério de Schwarz (SC);

$(* * *)$ Rejeita-se a presença de raiz unitária ao nível de $1 \%$.

Antes de verificar se as séries apresentam relações de causalidade por meio do teste de Toda e Yamamoto (1995), o próximo passo consiste em especificar um modelo VAR adequado, a partir da escolha do número correto de defasagens e quanto à inclusão de termos determinísticos, quais sejam: constante, tendência ou dummy.

A análise dos resultados da Tabela 2 indica que o modelo VAR deve conter uma ou duas defasagens, variando de acordo com o critério de informação observado. Neste estudo, optou-se pela inclusão de duas defasagens, conforme indicado pela maioria dos critérios (LR, FPE e AIC), pois acredita-se que duas defasagens reflitam de forma mais adequada a dinâmica de relacionamento entre as variáveis econômicas, visto que as decisões de investimento são baseadas em um período mais longo de tempo.

Quanto à inclusão de termos determinísticos, verifica-se que o modelo a ser estimado deve conter a variável dummy, que foi significativa a 5\% para o vetor que tem IDE como variável dependente. ${ }^{5}$ Seu coeficiente positivo indica que, a partir de 1990, houve mudança no padrão de entrada de investimentos diretos na economia, com aumento na taxa de crescimento do IDE, conforme esperado.

5 Os resultados do modelo VAR estimado encontram-se na Tabela A1 do Anexo. 
Tabela 2 - Definição do número de defasagens do modelo VAR

\begin{tabular}{cccccc}
\hline Defasagens & LR & FPE & AIC & SC & HQ \\
\hline 1 & -- & $1,07 \mathrm{e}-05$ & $-2,9303$ & $-2,6134^{*}$ & $-2,8066^{*}$ \\
2 & $19,3550^{*}$ & $1,01 \mathrm{e}-05^{*}$ & $-2,9904^{*}$ & $-2,3566$ & $-2,7430$ \\
3 & 11,0323 & $1,11 \mathrm{e}-05$ & $-2,9059$ & $-1,9552$ & $-2,5348$ \\
4 & 12,7691 & $1,15 \mathrm{e}-05$ & $-2,8725$ & $-1,6049$ & $-2,3777$ \\
\hline
\end{tabular}

Fonte: Elaboração própria a partir dos resultados da pesquisa.

Nota: (*) indica o número de defasagens ótimo de acordo com cada critério de informação: LR: Likelihood Ratio; FPE: Final Prediction Error; AIC: Akaike Information Criterion; SC: Schwarz Criterion; HQ: Hannan Quinn.

Finalmente, após a determinação da ordem de integração das variáveis e número ótimo de defasagens, estimou-se um VAR aumentado de ordem $(2+0)$ e, neste contexto, foi realizado o teste de Toda e Yamamoto para verificar a existência de relação causal no sentido de Granger entre as variáveis estudadas. Os resultados estão disponíveis na Tabela 3.

Tabela 3 - Teste de Toda-Yamamoto

\begin{tabular}{ccc}
\hline Variáveis & Qui-quadrado & Probabilidade \\
\hline Variável dependente: PIB & & \\
EXP $^{1}$ & 0,5213 & 0,4703 \\
IDE $^{2}$ & 0,3320 & 0,5645 \\
\hline Variável dependente: EXP & & \\
PIB & 6,3685 & 0,0116 \\
IDE & 0,7545 & 0,3850 \\
\hline Variável dependente: IDE & & \\
EXP & 0,6890 & 0,4065 \\
\hline
\end{tabular}

Fonte: Elaboração própria a partir dos resultados da pesquisa.

Nota: (1) A hipótese nula testada: EXP não causa (no sentido de Granger) PIB. (2) A hipótese nula testada: IDE não causa (no sentido de Granger) PIB. Analogamente, são testadas hipóteses de causalidade para as outras duas variáveis dependentes.

Após a estimação do modelo VAR com duas defasagens e variável dummy, foi realizado o teste LM (multiplicador de Lagrange) para verificar a presença de 
autocorrelação nos resíduos. Segundo resultado apresentado, não se pode rejeitar a hipótese de ausência de autocorrelação serial ${ }^{6}$.

A análise da Tabela 3 indica rejeição da hipótese nula de que o PIB não causa EXP, no sentido de Granger, e que EXP não causa (por Granger) IDE7 . Assim, conclui-se que PIB causa EXP, que por sua vez causa IDE. Logo, é possível inferir que o crescimento econômico afeta indiretamente o investimento direto estrangeiro por meio de seus efeitos sobre a exportação e que esta última funcionou como um intermediário ou "mecanismo de efeito", no período analisado.

Conforme a maioria dos estudos citados anteriormente, que confirmam a hipótese de export-led growth e/ou growth-driven export, o resultado deste estudo converge no que diz respeito à segunda hipótese, em que o crescimento econômico conduz à elevação das exportações por meio de causalidade direta entre as mesmas.

$O$ fato de as modificações no PIB serem desencadeadoras de efeitos nas demais variáveis pode ser atribuído ao fato de que o crescimento da demanda doméstica não acompanhou o aumento na produção, permitindo que produto disponível pudesse ser exportado e absorvido pelo mercado externo. Ou ainda, o crescimento da produção pode ser predominantemente de produtos tradable. No que diz respeito à relação causal no sentido de EXP para IDE, pode-se dizer que os fatores que contribuem para a elevação das exportações e diminuem as restrições ao livre comércio propiciaram clima favorável à entrada de IDE.

A ausência de relação direta entre IDE e PIB converge com os resultados apurados por Fernandes (2006), entre 1970 e 2003, por meio do teste de Toda e Yamamoto. A autora justificou a ausência de causalidade pela falta de condições que tornassem o IDE capaz de promover o crescimento. Neste caso, no entanto, verifica-se que é o crescimento econômico que indiretamente, por meio do crescimento das exportações, tem efeitos sobre os investimentos.

Adicionalmente, dada a existência de relações de causalidade verificadas entre as variáveis, torna-se oportuno o exame da Decomposição da Variância e das Funções Impulso-Resposta (FIR's). Para tanto, foi utilizado o método de decomposição de Cholesky. ${ }^{8}$ Tal método demanda que as variáveis sejam ordenadas no sentido da mais exógena para a mais endógena, de modo que as variáveis foram ordenadas como segue: PIB, EXP e IDE.

A partir da análise da Decomposição da Variância, é possível inferir qual proporção da variação de uma série pode ser explicada por choques nela mesma e em outras variáveis (ENDERS, 2004). Deste modo, conforme apresenta a Tabela 4, a variância do PIB é explicada em grande proporção por choques dado nele mesmo, não havendo reação em grande medida a choques nas demais variáveis.

6 Ver Tabela A1.

7 A partir deste ponto, onde se lê PIB, EXP e IDE deve-se entender que se referem à taxa variação das mesmas.

$8 \quad$ Para mais detalhes, ver Enders (2004). 


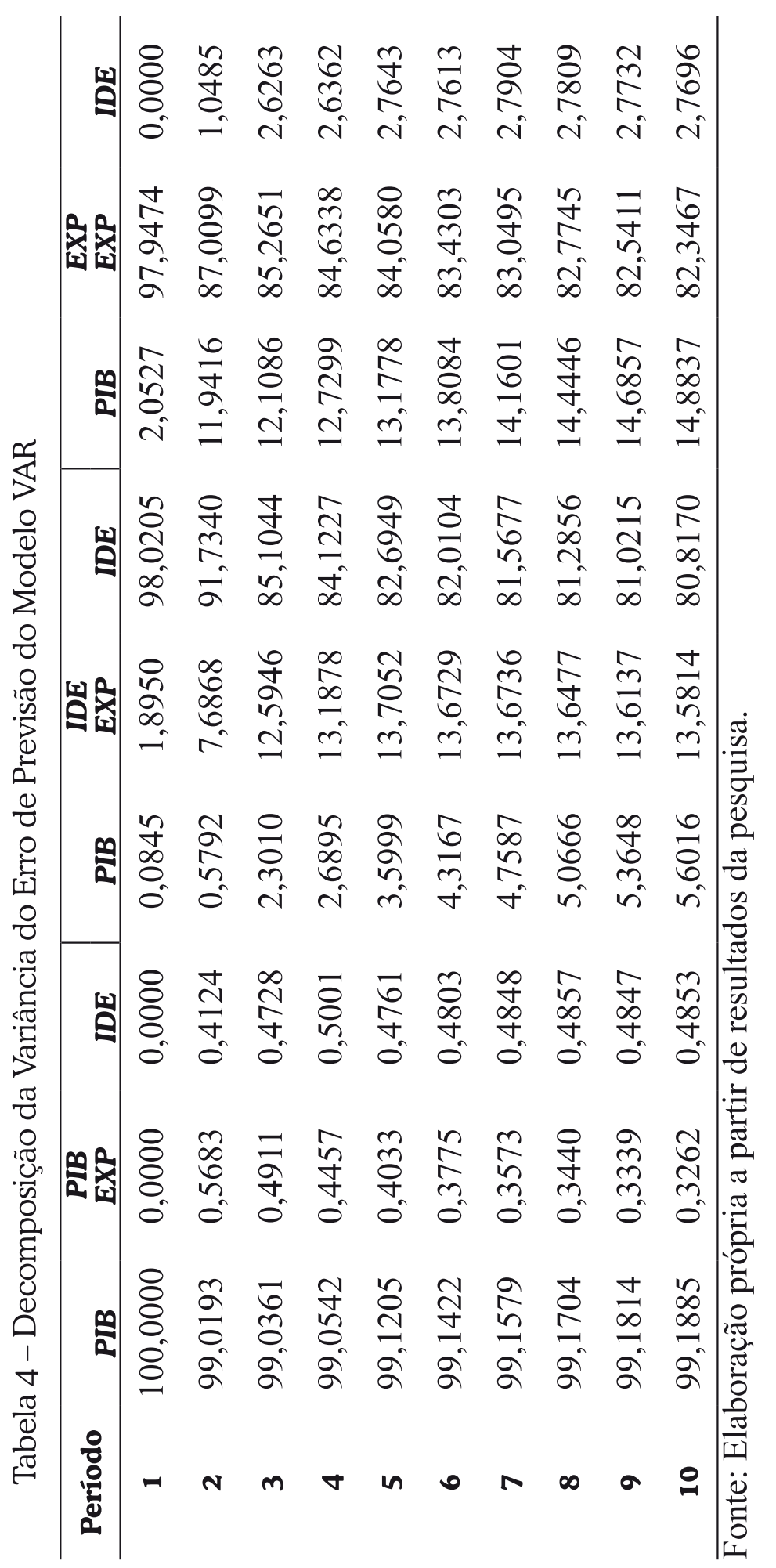


Quanto às exportações, é possível inferir que no primeiro ano sua variância é explicada em sua quase totalidade por ela mesma, mas, já no segundo ano, $12 \%$ de sua variância é explicada por choques no PIB, evoluindo até 15\% no décimo ano. Tal resultado corrobora a causalidade encontrada pelo teste de Toda e Yamamoto.

Com relação ao IDE, verifica-se que, no primeiro ano, sua variância é explicada em sua quase totalidade por ela mesma, já no segundo ano, choques nas exportações explicam 7\% de sua variância e, a partir do quarto ano, passam a explicar aproximadamente 13\% da variância do IDE, mantendo-se estável pelos próximos 6 anos. Este último resultado também vai ao encontro do resultado do teste de causalidade.

As FIR's medem a resposta das variáveis incluídas no VAR a choques exógenos sobre uma das variáveis do modelo, ao longo do tempo. A análise da Figura 1 (e) permite inferir que o efeito máximo nas exportações como resposta ao choque no PIB ocorre entre o primeiro e segundo ano, o que evidencia que as exportações reagem de forma rápida às mudanças no PIB. Além disso, o choque não se dissipa em um período de 10 anos. O comportamento do IDE se diferencia um pouco, pois, dado um choque em EXP, conforme análise da Figura 1(d) percebe-se que o maior efeito ocorre entre o segundo e terceiro ano e que o IDE retorna a sua tendência de longo prazo após sete anos. 
Figura 1 - Funções de Impulso-Resposta

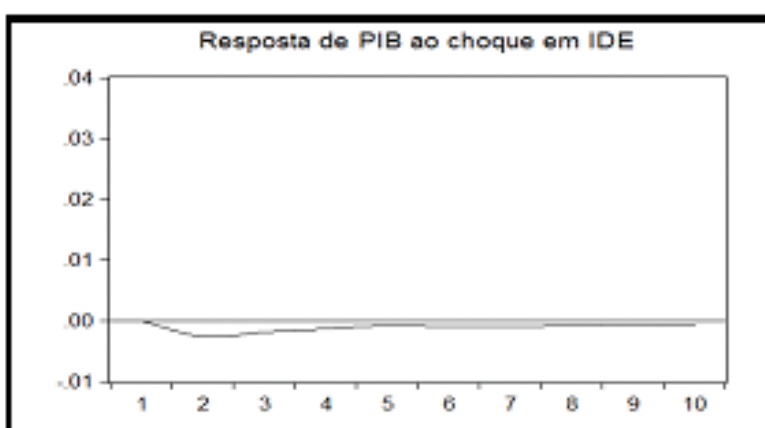

(a)

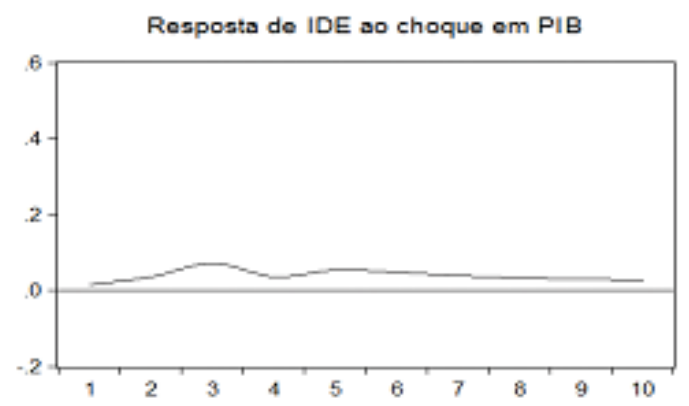

(c)

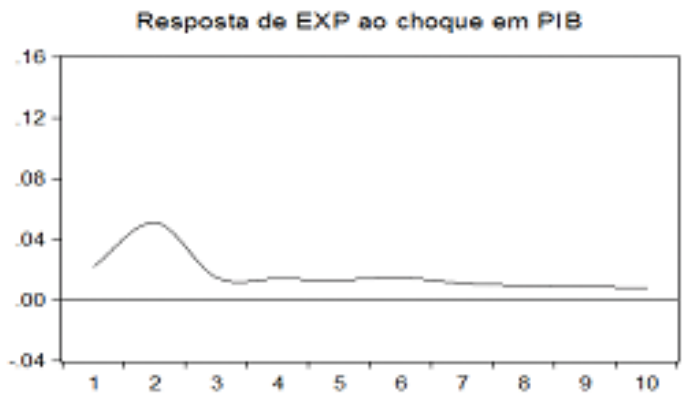

(e)

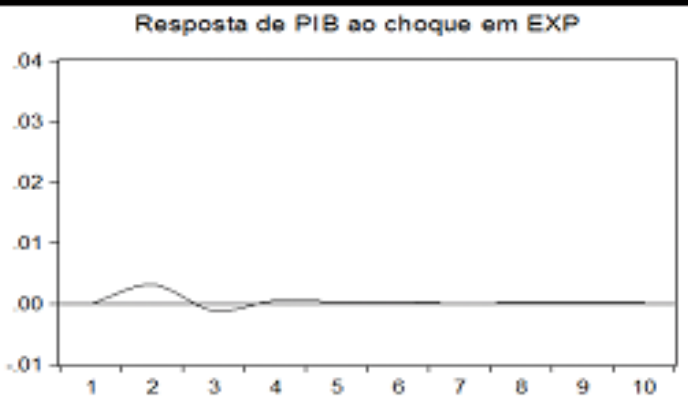

(b)

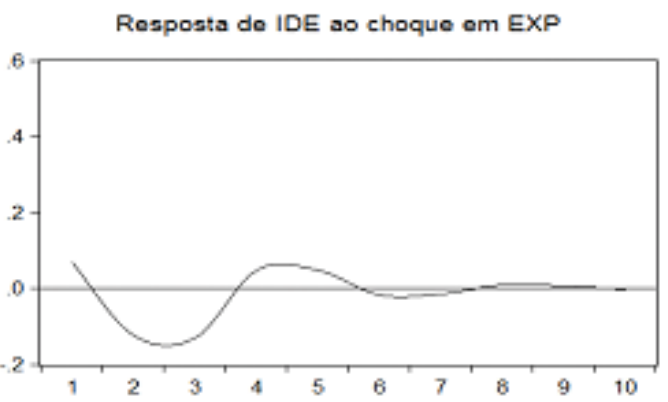

(d)

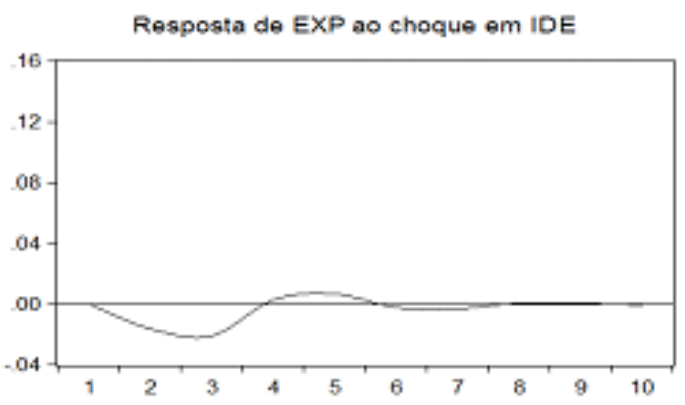

(n)

Fonte: Elaboração própria a partir dos resultados da pesquisa.

\section{Considerações Finais}

O contexto de recuperação da atividade econômica desencadeado nas duas últimas décadas foi o responsável pela motivação de verificar empiricamente as relações de interdependência entre crescimento, exportações e investimento direto estrangeiro, com vistas a contribuir com a literatura por meio da tentativa de verificar se alguma destas variáveis funcionaria como "mecanismo de efeito", pelo qual uma variável afetaria outra.

A análise dos resultados identificou que as exportações funcionam como um "mecanismo de efeito" pelo qual o crescimento econômico afeta o investimento 
direto estrangeiro de forma indireta. Foi possível verificar que as variáveis EXP e $I D E$ respondem rapidamente ao choque dado em PIB e EXP, respectivamente.

Se as relações de causalidade verificadas se mantiverem, acredita-se que, em primeiro lugar, deve-se fomentar políticas de incentivo ao crescimento da produção, visto que esta é desencadeadora das relações que culminam com a entrada de investimentos diretos estrangeiros. Ademais, condições para que as exportações possam intermediar os efeitos entre crescimento e IDE devem ser promovidas, fazendo com que o aumento das exportações resulte, de fato, em aumento dos investimentos. Assim, deve-se incentivar, com investimentos em ganhos de competitividade, o crescimento das exportações, dada sua relação direta com os investimentos diretos estrangeiros.

Em síntese, os resultados apontam que a ação do poder público deve ser direcionada no sentido de adequar a infraestrutura brasileira à criação de condições que potencializem não só o aumento do nível do PIB, do fluxo de exportações e da captação de IDE, mas também beneficie a ocorrência dos efeitos indiretos, evidenciados neste trabalho, entre as variáveis consideradas.

\section{Referências}

AWOKUSE, T. O. Is the Export-Led Growth Hypothesis Valid for Canada? Canadian Journal of Economics, Toronto, CA, v. 36, n. 1, p. 126-136, Feb. 2003.

BANCO CENTRAL DO BRASIL. Relatório Anual 2010, v 46. 2011. Disponível em: < http:// www.bcb.gov.br/?BOLETIM2010 >. Acesso em: 23 abr. 2011.

BONELLI, R.; PINHEIRO, A. C. Abertura e Crescimento Econômico no Brasil. In: GIAMBIAGI, F.; BARROS, O. (Org.). Brasil Globalizado. Rio de Janeiro: Elsevier, 2008.

BORENSTEIN, E. J. G.; LEE, J. W. How does Foreign Direct Investment Affect Economic Growth?: NBER Working Paper, Cambridge, MA, n. 5.057, Mar. 1995.

BUCKLEY, P. J.; CASSON, M. The Future of the Multinational Enterprise. London: Macmillian, 1976.

The Optimal Timing of a Foreign Direct Investment. Economic Journal, Cambridge, GB, v. 91, n. 361, p. 75-87, Mar. 1981.

BUCKLEY, P. J. et al. FDI, Regional Differences and Economic Growth: Panel Data Evidence from China. Transnational Corporations, New York, US, v. 11, n. 1, p. 1-28, Apr. 2002.

BUENO, R. L. S. Econometria de Séries Temporais. São Paulo: Cengage, 2008.

CHO, K. Studies on Knowledge Spillovers, Trade, and Foreign Direct Investment: Theory and Empirics. Thesis (Doctorate) - Department of Economics, University of Colorado, Boulder, $\mathrm{CO}, 2005$.

CHOW, P. C. Y. Causality between export growth and industrial development: Empirical evidence from the NICs. Journal of Development Economics, Amsterdam, NL, v. 26, n. 1, p. 55-63, Jun. 1987. 
DRITSAKI, M.; DRITSAKI, C.; ADAMOPOULOS, A. A Causal Relationship between Trade, Foreign Direct Investment, and Economic Growth for Greece. American Journal of Applied Sciences, v. 1, n. 3, p. 230-235, 2004.

DUNNING, J. H. Explaining international production. London: Unwin Hyman, 1988.

ENDERS, W. Applies econometric time series. $2^{\text {nd }}$ ed. Hoboken: John Wiley, 2004.

FASANO FILHO, U. A Expansão das Exportações e o Crescimento Econômico: O Caso do Brasil, 1969-84. Revista Brasileira de Economia, Rio de Janeiro, v. 42, n. 1, p. 73-81, out. 1988.

FERNANDES, E. A. Investimento Direto Externo no Brasil: Efeitos no Crescimento, nas Exportações e no Emprego. Tese (Doutorado) - Departamento de Economia Rural, Universidade Federal de Viçosa, Viçosa, 2006.

FOCHEZATTO, A.; KOSHIYAMA, D.; ALENCASTRO, D. Testando Relações de Causalidade entre Comércio Externo e Crescimento Econômico em Países da América Latina: Evidências de Dados em Painel e Séries Temporais. Economia, Brasilia, v. 11, n. 3, p.597-629, set./dez. 2010.

FOCUS/BC. Desempenho do Comércio Exterior Brasileiro no Primeiro Semestre de 2007. Relatório do Banco Central do Brasil, 2007.

GRANGER, A. Investigating Casual Relationship by Econometric Models and Cross Spectral Methods. Econometrica, Chicago, US, v. 37, n. 3, p. 424-458, Aug. 1969.

GROSSMAN, G.; HELPMAN, E. Innovation and Growth in the Global Economy. Cambridge, MA: MIT Press, 1991.

HIRSCH, S. An International Trade and Investment Theory of the Firm. Oxford Economic Papers, Oxford, GB, v. 28, n. 2, p. 258-270, Jul. 1976.

HSIAO, F. S. T.; HSIAO, MEI-CHU W. FDI, Exports and GDP, in East Southeast Asia - Paine Data Versus Time - Series Causality Analyses. Journal of Asian Economics, Amsterdam, NL, v. 17, n. 6, p. 1082-1106, Dec. 2006.

JAYME JÚNIOR, F. G. Comércio Internacional e Crescimento Econômico: O Comércio Afeta o Desenvolvimento? Revista Brasileira de Comércio Exterior, Rio de Janeiro, ano 14, n. 69, out./dez. 2001.

KALDOR, N. The Case for Regional Policies. Scottish Journal of Political Economy, Harlow, GB, v. 17, n. 3, p. 337-48, Nov. 1970.

KALIRAJAN, K.; MIANKHEL, A.; THANGAVELU, S. Foreign Direct Investment, Exports, and Economic Growth in Selected Emerging Countries: Multivariate VAR analysis. Working Paper MPRA, Munich, n. 22763, 2009.

KEESING, D. B.; LALL, S. Marketing Manufactured Exports from Developing Countries: Learning Sequences and Public Support. In: HELLEINER, G. (Ed.). Trade Policy, Industrialization, and Development: New Perspectives. Oxford: Clarendon Press, 1992.

KÓNYA, L. Export-Led Growth or Growth-Driven Export? New Evidence Form Granger Causality Analysis on OECD Countries. Working Papers Central European University, Budapest, v. 15, Dec. 2000.

LACERDA, A. C. Empresas Transnacionais, Investimentos Diretos Estrangeiros e Exportações na Economia Brasileira na Década de Noventa. Economia Aplicada, São Paulo, v. 8, n. 4, p. 619-658, dez. 2004.

LIU, X.; WANG, C.; WEI, Y. Causal Links between Foreign Direct Investment and Trade in China. China Economic Review, Amsterdam, NL, v. 12, n. 2-3, p. 190-202, Summer 2001. 
LUCAS, R. E. On the mechanics of economic development. Journal of Monetary Economics, Amsterdam, NL, v. 22, n. 1, p. 3-42, 1988.

MATESCO, V. R.; LIMA, M. F. F.; DEANA, T. Fluxos Mundiais de Investimentos: A Internacionalização da Economia Brasileira. In: LACERDA, A. C. (Org.). Desnacionalização: Mitos, Riscos e Desafios. São Paulo: Contexto, 2000.

MATOS, O. C. Interrelations among Financial Development, Exports and Economic Growth: Analysis of the Brazilian Experience. Banco Central do Brasil Technical Notes, Brasília, v. 40, p. 1-39, Oct. 2003.

NAIR-REICHERT, U.; WEINHOLD, D. Causality Tests for Cross-Country Panels: New Look At FDI and Economic Growth in Developing Countries. Oxford Bulletin of Economics and Statistics, Oxford, GB, v. 63, n. 2, p. 153-171, May 2001.

NONNENBERG, M. J. B; MENDONÇA, M. J. C. Determinantes dos Investimentos Diretos Externos em Países em Desenvolvimento. Estudos Econômicos, São Paulo, v. 35, n. 4 , p. 631-655, out./dez. 2005.

ROMER, P. Increasing Returns and Long-Run Growth. Journal of Political Economy, Chicago, US, v. 94, n. 5, p. 1002-1037, Oct.1986.

RUGMAN, A.; DONALD, J. L.; LAURENCE, D. International Business: Firm and Environment. New York: McGraw-Hill, 1985.

SILVA, A. G.; VIRGOLINO, J. R. O.; LIMA, R. C. A Lei de Thirlwall e a Economia Brasileira: Uma Breve Consideração. Revista Econômica do Nordeste, Fortaleza, v. 31, n. 9, p. 852-864, nov. 2000.

SILVA, I. E. M; LIMA, R. C.; BEZERRA, J. F. Exportações e Produtividade: Teste à Teoria do Crescimento Liderado pelas Exportações para a Indústria de Transformação do Brasil. In: CONGRESSO DA SOCIEDADE BRASILEIRA DE ECONOMIA, ADMINISTRAÇÃO DE SOCIOLOGIA RURAL, 48., 2010, Recife. Anais... Recife: SOBER, 2010.

SOLOW, R. M. A Contribution to the Theory of Economic Growth. Quarterly Journal of Economics, Cambridge, US, v. 70, p. 155-173, Feb. 1956.

TODA, H. Y.; YAMAMOTO, T. Statistical Inferences in Vector Autoregressions with Possibly Integrated Processes. Journal of Econometrics, Amsterdam, NL, v. 66, n. 1-2, p. 225-250, Mar. /Apr. 1995.

TYLER, W. G. Substituição de Importações e Expansão das Exportações como Fontes de Crescimento Industrial no Brasil. Estudos Econômicos, São Paulo, v. 12, n. 3, 1982.

ZAPATA, H. O.; RAMBALDI, A. N. Monte Carlo Evidence on Cointegration and Causation. Oxford Bulletin of Economics and Statistics, Oxford, GB, v. 59, n. 2. p. 285-298, 1997.

ZHANG, Q.; FELMINGHAN, B. The Relationship between Inward Direct Investment in China's Provincial Export Trade. China Economic Review, Amsterdam, NL, v. 12, n. 1, p. 82-99, 2001. 


\section{Apêndice A - Estimativa do Modelo VAR}

\begin{tabular}{|c|c|c|c|c|c|}
\hline Equação & Variável Dependente & Variável & Coeficiente & \multicolumn{2}{|c|}{ Desvio-padrão } \\
\hline \multirow{8}{*}{1} & $P I B_{t}$ & $P I B_{t-1}$ & 0,4886 & \multicolumn{2}{|c|}{$-0,1315$} \\
\hline & & $\mathrm{PIB}_{\mathrm{t}-2}$ & 0,3758 & \multicolumn{2}{|c|}{$-0,1286$} \\
\hline & & $I D E_{t-1}$ & $-0,0054$ & \multicolumn{2}{|c|}{$-0,00935$} \\
\hline & & $I D E_{t-2}$ & $-0,0001$ & \multicolumn{2}{|c|}{$-0,00917$} \\
\hline & & $E X P_{t-1}$ & 0,0230 & \multicolumn{2}{|c|}{$-0,03188$} \\
\hline & & $\operatorname{EXP}_{t-2}$ & $-0,0233$ & \multicolumn{2}{|c|}{$-0,0327$} \\
\hline & & DUMMY & 0,0097 & \multicolumn{2}{|c|}{$-0,00854$} \\
\hline & \multicolumn{2}{|c|}{$\mathrm{R}^{2}=0,2571$} & \multicolumn{3}{|c|}{ Estatística $\mathrm{F}=3,1146$} \\
\hline \multirow{8}{*}{2} & $I D E_{t}$ & $P I B_{t-1}$ & 1,4764 & \multicolumn{2}{|c|}{$-1,7786$} \\
\hline & & $P I B_{t-2}$ & 2,9042 & \multicolumn{2}{|c|}{$-1,7393$} \\
\hline & & $I D E_{t-1}$ & 0,0682 & \multicolumn{2}{|c|}{$-0,12648$} \\
\hline & & $I D E_{t-2}$ & $-0,2483$ & \multicolumn{2}{|c|}{$-0,12404$} \\
\hline & & $E X P_{t-1}$ & $-0,8592$ & \multicolumn{2}{|c|}{$-0,43113$} \\
\hline & & $E X P_{t-2}$ & $-0,6910$ & \multicolumn{2}{|c|}{$-0,44225$} \\
\hline & & DUMMY & 0,2424 & \multicolumn{2}{|c|}{$-0,11553$} \\
\hline & \multicolumn{2}{|c|}{$\mathrm{R}^{2}=0,2158$} & \multicolumn{3}{|c|}{ Estatística $F=2,4766$} \\
\hline \multirow{8}{*}{3} & $E X P_{t}$ & $P I B_{t-1}$ & 1,3803 & \multicolumn{2}{|c|}{$-0,5470$} \\
\hline & & $P I B_{t-2}$ & $-0,2067$ & \multicolumn{2}{|c|}{$-0,5349$} \\
\hline & & $I D E_{t-1}$ & $-0,0338$ & \multicolumn{2}{|c|}{$-0,0389$} \\
\hline & & $I D E_{t-2}$ & $-0,0313$ & \multicolumn{2}{|c|}{$-0,03814$} \\
\hline & & $E X P_{t-1}$ & 0,0646 & \multicolumn{2}{|c|}{$-0,13258$} \\
\hline & & $E X P_{t-2}$ & $-0,2229$ & \multicolumn{2}{|c|}{$-0,136$} \\
\hline & & DUMMY & 0,0400 & \multicolumn{2}{|c|}{$-0,03553$} \\
\hline & \multicolumn{2}{|c|}{$\mathrm{R}^{2}=0,1080$} & \multicolumn{3}{|c|}{ Estatística $\mathrm{F}=1,0894$} \\
\hline \multirow{3}{*}{\multicolumn{2}{|c|}{ Autocorrelação (LM-teste) }} & Defasagem & Estatística d & Teste & p-valor \\
\hline & & $1^{\mathrm{a}}$ & 12,2795 & & 0,1980 \\
\hline & & $2^{\mathrm{a}}$ & 12,6097 & & 0,1811 \\
\hline
\end{tabular}

Fonte: Elaboração própria a partir dos resultados da pesquisa.

Recebido em: 04/09/2011. Aceito em: 16/03/2012. 